\title{
Espacios rupestres como «obras abiertas»: una mirada a los procesos de confección y transformación de los abrigos con arte rupestre del este de Catamarca (Argentina)
}

\author{
Lucas I. GHeCo*, Marcos N. QuesAdA*, Gabriel YBARRA*, \\ Andrea PoliszuK ${ }^{* *}$ y Omar Burgos*** \\ * CONICET - Escuela de Arqueología, Universidad Nacional de Catamarca (Argentina) \\ ** Instituto Nacional de Tecnología Industrial (Argentina) \\ *** Grupo Argentino del Color (Argentina) \\ gheco@hotmail.com mkesada@yahoo.com.argabriel@inti.gob.ar \\ ankapoli@inti.gob.ar omar.burgos2@gmail.com
}

Recibido: 16 de agosto de 2012

Aceptado: 29 de abril de 2013

\section{RESUMEN}

En este trabajo intentamos avanzar en el conocimiento del proceso de pintado de las cuevas con arte rupestre de la Sierra de Ancasti (Provincia de Catamarca, Argentina) a partir del análisis químico de las mezclas pigmentarias con las que fueron realizadas las pinturas. De este modo, planteamos la posibilidad de comprender algunos de estos abrigos pintados como «obras abiertas», es decir, como el resultado del agregado de motivos a lo largo de un proceso cuya duración, quizás larga, aún no conocemos.

Palabras clave: Arte rupestre, Sierra de Ancasti, Oyola, Arqueología.

Rock Spaces as «Open Works»: A Look at the Process of Making and Transforming Caves with Rock Art in the East of Catamarca (Argentina)

\section{ABSTRACT}

In this paper we attempt to advance the understanding of the process of painting in caves with rock art at Ancasti's Highlands (Catamarca Province, Argentina) from chemical analysis of pigment mixtures with which the paintings have been made. Thus, we suggest the possibility of understanding some of the art in these caves as «open works», i.e.: as the result of addition of motives over a process whose duration, perhaps long, we do not know.

Key words: Rock art, Highlands of El Alto-Ancasti, Oyola, Archaeology.

Sumario: 1. Introducción. 2. La Sierra de Ancasti. 3. Oyola. 4. Análisis químicos. 5. Palabras finales. 6. Referencias bibliográficas

\section{Introducción}

A mediados del siglo pasado, Umberto Eco (1992 [1962]) utilizaba el concepto de obra abierta para hacer referencia a una serie de composiciones musicales que otorgaban una particular autonomía ejecutiva al intérprete, quien podía intervenir en la forma de la composición de acuerdo con su particular sensibilidad. Con esto, Eco no intentaba marcar una contraposición entre obras «abiertas» y «cerradas», sino destacar que todas las obras de arte, hasta las más tradicionales, se presentan como un campo de posibilidades para quien las ejecuta y/o aprecia. De este modo, el movimiento 
musical que dejaba cierta libertad creativa al intérprete era sólo el reconocimiento consciente de un proceso general descrito por el autor en los siguientes términos:

«una obra de arte es un objeto producido por un autor que organiza una trama de efectos comunicativos de modo que cada posible usuario pueda comprender (...) la obra misma, la forma originaria imaginada por el autor (...) no obstante (...) cada usuario tiene una concreta situación existencial, una sensibilidad particularmente condicionada (...) de modo que la comprensión de la forma originaria se lleva a cabo según determinada perspectiva individual. (...) En tal sentido, pues, una obra de arte, forma completa y cerrada en su perfección de organismo perfectamente calibrado, es asimismo abierta, posibilidad de ser interpretada de mil modos diversos sin que su irreproducible singularidad resulte por ello alterada» (Eco 1992: 30).

Es decir, mediante el concepto de obra abierta, Eco pretende hacer referencia a dos características de toda obra de arte: que puede ser modificada y que puede ser interpretada de varias maneras más allá de la intención del autor original. De este modo, la apertura de las obras de arte se plantea en dos sentidos. Por un lado, la polisemia de lecturas según cada perspectiva individual; por el otro, el carácter no acabado de cualquier manifestación artística.

Sin olvidar la distancia que separa la concepción occidental moderna del arte de las manifestaciones pictóricas prehispánicas objeto de esta investigación, el concepto de Eco resulta de gran valor para intentar aproximarnos a los procesos históricos de construcción y transformación de los abrigos con arte rupestre del Este de la Provincia de Catamarca, Argentina. En las próximas líneas intentaremos explicar por qué.

\section{La Sierra de Ancasti}

La Sierra de El Alto-Ancasti ${ }^{1}$ se dispone en el sector oriental de la Provincia de Catamarca (Argentina) y a escasos kilómetros del límite interprovincial con la jurisdicción de Santiago del Estero (Figura 1). Este cordón integra el sistema geográfico-geológico de las Sierras Pampeanas Septentrionales y presenta una forma elongada en sentido Norte-Sur. Su longitud aproximada es de $170 \mathrm{Km}$ desde Alijilán, al Norte, hasta Casa de Piedra, al Sur. Su ancho, en la latitud de la ciudad de Catamarca, es de $40 \mathrm{Km}$. y sus máximas alturas oscilan en torno a los 2.000 m.s.n.m. (Aceñolaza et al. 1983).

Desde mediados del siglo XX, diversos investigadores se dedicaron al estudio de numerosos sitios con arte rupestre dispuestos en la ladera oriental de la sierra, como La Tunita, La Candelaria o Cueva de la Salamanca, Campo de las Piedras, Ampolla, Inasillo, Oyola, Los Algarrobales, La Toma, El Lechico, etc. (De la Fuente 1969, 1979a, 1979b, 1990; De la Fuente y Díaz Romero 1974, 1979; De la Fuente, Nazar y Pelli 2005; De la Fuente, Tapia y Reales 1982, 1983; Gómez y De la Fuente 1989; Gheco 2011; González 1977; Gordillo y Calomino 2010; Gramajo y Martínez Moreno 1978; Llamazares 1993, 1999-2000; Nazar 2003a, 2003b, 2010; Nazar y De la Fuente 2009, Nazar et al. 2010; Quesada y Gheco 2011; Segura 1970). En su mayo-

1 Siglos atrás conocida como Sierra de Santiago (Segura 1988) y en la actualidad denominada comúnmente como Sierra de Ancasti. 
ría, atribuyeron las pinturas rupestres a la Cultura de La Aguada ${ }^{2}$ (ca. 600-900 d.C.) de acuerdo con las similitudes estilísticas apreciadas entre algunas de las pictografías y los motivos definidos como pertenecientes a la cerámica de dicha cultura (por ejemplo: De la Fuente 1969; De la Fuente y Arrigoni 1975; González 1977, 1998; Gramajo 2001; Llamazares 1999-2000; Segura 1988).

El énfasis en la descripción de los motivos adscritos a Aguada creó, con el correr del tiempo, una imagen un tanto homogénea del arte rupestre de la Sierra de Ancasti como «arte Aguada». Sin embargo, una lectura detallada de los trabajos de los mismos investigadores nos permite advertir que, al tiempo que atribuían muchas de las pinturas a esta cultura, también destacaban que algunos motivos presentaban características que los apartaban de los diseños típicos de ella. Así, los consideraron como pertenecientes a otros momentos o períodos, otras culturas o como el resultado de

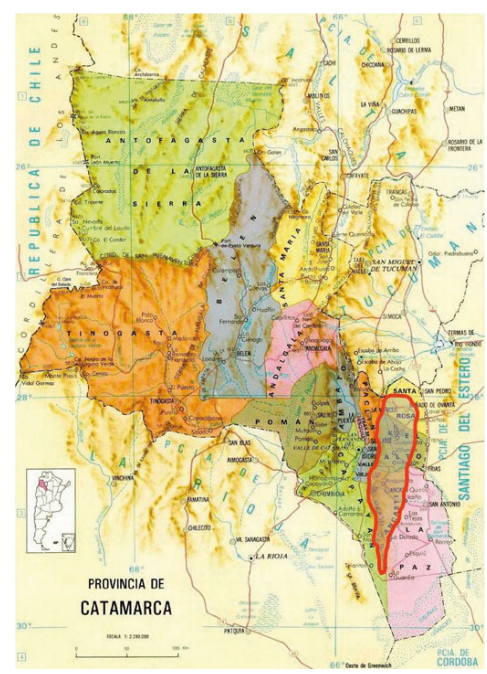

Figura 1: Mapa de la Provincia de Catamarca y ubicación de la Sierra de El Alto-Ancasti. influencias de pueblos de otras regiones.

En nuestras investigaciones en los sitios con arte rupestre de la sierra, pudimos observar una serie de indicios que nos permiten sospechar que algunas cuevas y aleros con arte, antes que conjuntos elaborados en un único evento de pintado, son el resultado de sucesivos agregados de figuras en una secuencia temporal cuya duración desconocemos. Las diferencias estilísticas entre motivos de distintos sitios, la diversidad de colores y disposiciones espaciales de las pinturas en el interior de los abrigos, sumadas al hallazgo preliminar de superposiciones entre figuras, fueron algunas de las características que nos permitieron arribar a esta intuición. Sin embargo, no se trata de una idea del todo original, sino que retoma un conjunto de valiosas observaciones de los investigadores que trabajaron en la zona, quienes esbozaron este problema al plantear la posibilidad de que algunos motivos rupestres fueran el resultado de la acción de diferentes sociedades o culturas (De la Fuente, Tapia y Reales 1982, 1983; González 1977; Gramajo y Martínez Moreno 1978; Segura 1970). La intención de esta investigación es continuar estas ideas, pero ya no para evaluar la existencia o temporalidad de las diferentes culturas a las que se vinculó el arte rupestre, sino para aproximarnos a los complejos procesos de pintado que fueron construyendo y modificando los abrigos con arte rupestre. En este trabajo intentamos comenzar a describir estos procesos históricos y buscar alternativas técnicas que nos permitan acceder a su conocimiento. Para ello, retomamos parte de los resultados de un estudio donde planteamos el análisis intensivo de un abrigo con arte rupestre (Oyola 7) del sitio arqueológico de Oyola (Dpto. El Alto, Prov. Catamarca) a partir del relevamiento en detalle de cada uno de los motivos y las superposiciones entre figuras y el análisis químico

2 Para mayor información sobre la Cultura de La Aguada, ver González 1961-64 y 1998. 




Figura 2: Ubicación de algunos de los sitios con arte rupestre de la Sierra de El Alto-Ancasti.

de las mezclas pigmentarias con que fueron confeccionadas las pinturas (Quesada, Gheco, Burgos et al. 2011). En las secciones siguientes presentamos los resultados de la aplicación de dos técnicas de análisis químico (microscopía electrónica de barrido con análisis elemental SEM-EDS y FTIR) para avanzar en el conocimiento del proceso de pintado de Oyola 7.

\section{Oyola}

A pocos kilómetros de la población de Vilismán, en el Departamento El Alto de la Sierra de Ancasti, se encuentra la localidad de Oyola (Figura 2). Allí, a no más de unos cientos de metros del camino principal (Ruta Provincial $\mathrm{N}^{\circ} 103$ ), se disponen una serie de cuevas y aleros rocosos con arte rupestre declarados Patrimonio Cultural de la Provincia de Catamarca en 2002 (Ley 5081/2002).

El sitio arqueológico de Oyola se dispone en el sector Noreste de un gran batolito o plutón de unos 2,5 km de diámetro, identificado en la bibliografía especializada con el nombre de la localidad de Vilismán (Aceñolaza et al. 1983). La erosión de miles de años moldeó en un conjunto de grandes rocas de esta intrusión granítica distintas cámaras y oquedades, algunas de la cuales fueron utilizadas desde tiempos prehispánicos para plasmar pinturas en sus paredes y techos. Desde hace algunos años continuamos con el relevamiento del sitio iniciado hacia la década de 1970 por Amalia Gramajo y Hugo Martínez Moreno (1978). Hasta el momento, hemos podido diferenciar, al menos, diecisiete abrigos con manifestaciones rupestres.

La Cueva 7 de Oyola se dispone en la base de una gran roca granítica en la cima de una lomada adyacente al poblado actual de Oyola. En su interior, diversas manifestaciones pintadas y grabadas, realizadas sobre sus paredes y techos, se alternan y superponen (Figura 3). La cueva posee dos accesos o bocas en sus extremos. Al Oeste, la entrada tiene un escaso tamaño y se abre hacia un gran playón pétreo desde donde se domina la visión del paisaje circundante. Por el contrario, la boca Este de la cueva presenta mayores dimensiones y se dispone a pocos metros (15 m aprox.) de la Cueva 6. La gran vegetación que recubre esta abertura y la proximidad de otras rocas hacen muy difícil la visión y el acceso a través de ella. 
Figura 3: Vista del interior de la Cueva 7 de Oyola. Fotografía: Oscar Dechiara.

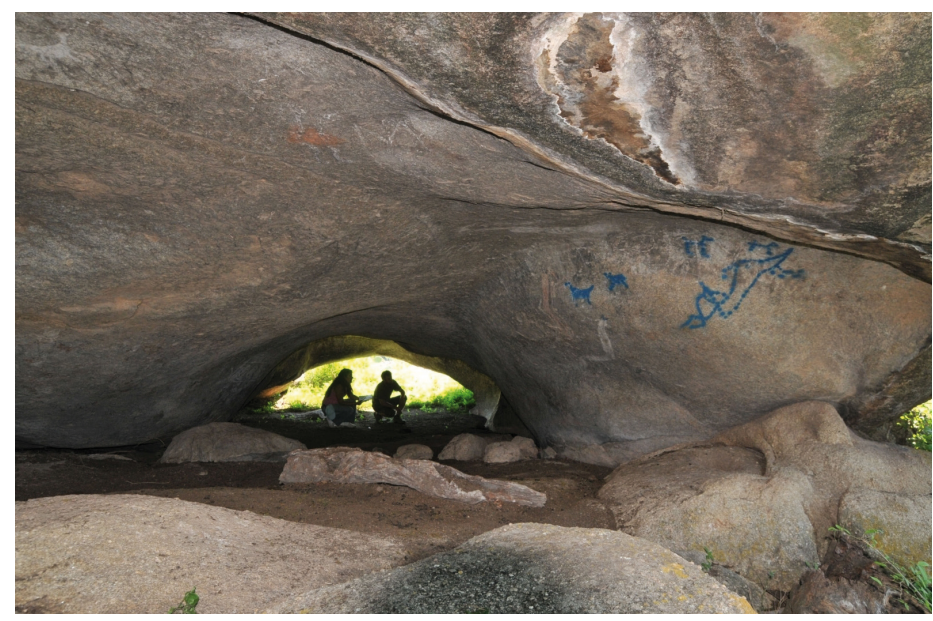

La cueva presenta una forma longitudinal en sentido Este-Oeste. El interior es relativamente grande en relación con las demás cuevas pintadas (aproximadamente $1,6 \mathrm{~m}$ de altura, $5 \mathrm{~m}$ de largo, $2 \mathrm{~m}$ de ancho), disminuyendo su altura en algunos sectores. Su tamaño permite la permanencia simultánea de varias personas observando los motivos de arte rupestre. La intensidad de la luz natural que accede a la cueva es muy escasa, aunque hace posible la observación de los motivos durante gran parte del día. El piso desciende en sentido Este-Oeste y se encuentra recubierto de guano de distintos animales.

En el exterior de la cueva, en el espacio próximo al acceso Oeste, se han hallado algunos pequeños fragmentos de cerámica sin decoración y un mortero tallado sobre una roca horizontal. Junto con un mortero en el interior de la cueva y las pinturas propiamente dichas, éstos son los únicos vestigios materiales localizados.

Las manifestaciones rupestres prehispánicas en el interior de esta cueva han sido confeccionadas mediante la pintura en colores cremas, negros y rojos. Para algunas, según Gramajo y Martínez Moreno (1978), se realizó un previo raspado de la roca sobre el que luego se pintó. Los motivos modernos, por su parte, fueron raspados o pintados con aerosol azul. En total, se han detectado 75 motivos $^{3}$. En términos generales, se destacan por su alta frecuencia las figuras que interpretamos como camélidos en once oportunidades (Figura 4). Le siguen una serie de figuras geométricas cuya forma es similar a tres rectángulos verticales unidos por la base, siendo el central de mayor altura, en un número de nueve. También podemos apreciar varios motivos con rasgos antropomorfos (ocho) y se destaca por la escasa frecuencia en relación a los demás sitios de la Sierra de Ancasti la figura del jaguar (sólo un caso) ${ }^{4}$.

Como mencionamos, una serie de indicios nos permiten sospechar que algunos de los abrigos con arte rupestre de la Sierra de Ancasti, en este caso Oyola 7, son

3 Entre ellos, incluimos los grafitis e inscripciones modernas con la intención de documentar otros episodios de transformación de los repertorios pictóricos de la cueva. Con esto no pretendemos favorecer este tipo de prácticas en los sitios arqueológicos.

4 Para ver una descripción más detallada de los motivos de Oyola 7 y de las demás cuevas del sitio, ver Quesada, Gheco, Burgos et al. 2011. 


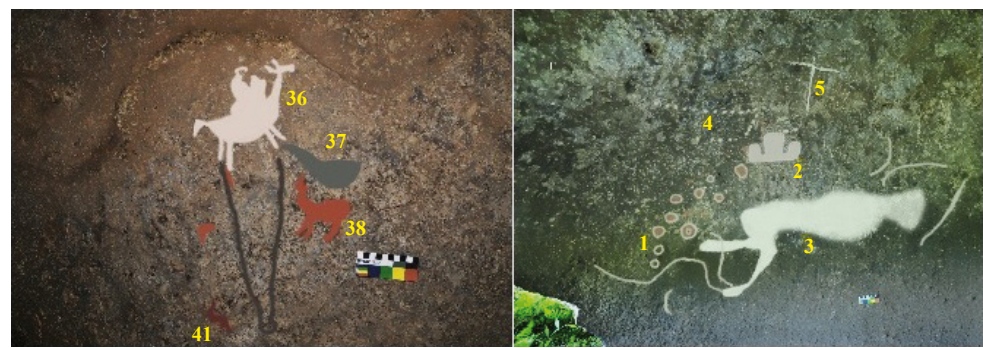

Figura 4: Calcos

digitales de algunos

motivos de arte

rupestre de Oyola 7.

el resultado, no acabado, de la estratificación de diferentes eventos de confección y modificación de sus repertorios pictóricos a lo largo del tiempo. Esta secuencia de múltiples transformaciones nos desafía a agudizar nuestras técnicas analíticas con el fin de intentar aproximarnos a las relaciones que estas modificaciones habrían tenido con las prácticas de las personas que realizaron y dieron sentido a las pinturas. Los problemas de conservación que afectan a esta cueva hacen aún más difícil esta tarea $^{5}$. Sin embargo, existen una serie de técnicas que, en su combinación, pueden permitirnos avanzar en esta dirección, entre ellas: el estudio de las superposiciones, los análisis químicos de las pinturas, la datación absoluta de los motivos, los análisis morfológicos de las figuras, la excavación de los pisos de las cuevas. A continuación presentamos los resultados de la aplicación de una de estas técnicas para el estudio del arte rupestre de la Cueva Oyola 7.

\section{Análisis químicos}

Una de las formas de indagar en los procesos de formación y transformación de los sitios con arte rupestre es a través del análisis químico de las mezclas pigmentarias con las que han sido realizadas las pinturas. Al hablar de mezclas pigmentarias nos referimos al producto de la mixtura de uno o varios sólidos pulverizados (pigmentos o cargas inertes) con un líquido (ligante) que contiene una sustancia filmógena que le da cohesión y adhesión a la composición (Babot y Apella 2010; Nazar et al. 2010). Bajo el concepto de pigmento se agrupan todas aquellas partículas o fragmentos minerales o rocosos que, por sus propiedades físicas, actúan como cromóforos (p.e. goethita, hematita, pirolusita, etc.). Éstos deben ser molidos y mezclados o dispersados con ciertos componentes (cargas y ligantes) que tienen la peculiaridad de otorgar particulares características a la composición: mejoran las propiedades adhesivas de las pinturas, cambian su textura y consistencia, proveen mejor cobertura y proporcionan mejor preservación. Algunos materiales -como arcilla, yeso, calcita, cuarzo, hueso, talco y feldespato potásico- le confieren cuerpo a la pintura, por lo que se emplean frecuentemente para actuar como carga; su utilización arqueológica ha sido

5 En Oyola 7 se detectaron problemas de conservación de origen antrópico y natural. Entre los primeros, se destaca la presencia de rayones próximos o sobre los motivos de arte rupestre. En cuanto a los factores naturales, la cueva brinda refugio a animales, por lo que se evidencia deterioro por frotación de ganado. Otros factores biológicos detectados son: presencia de nidos de avispas y abejas, líquenes, aves, acreciones en bordes de goteo (Granizo 2010). 
corroborada para el Noroeste Argentino (Aschero y Podestá 1986). Por su parte, los ligantes facilitan la integración de pigmentos y cargas y favorecen la adherencia de las mezclas a la superficie rocosa o soporte. Los aceites vegetales y las grasas animales, entre otros componentes de origen orgánico, fueron los ligantes por excelencia en las pinturas prehispánicas (Yacobaccio et al. 2008).

Usualmente, los análisis químicos de las mezclas pigmentarias fueron utilizados como vía de acceso al estudio de los materiales y técnicas empleados en la confección de los motivos de arte rupestre (Bonneau et al. 2012). Sin descuidar estos aspectos, en este trabajo intentamos destacar la importancia de estos estudios, sus posibilidades y límites para profundizar en el conocimiento del proceso de construcción de los espacios rupestres. Para ello, primero, debemos realizar algunos comentarios.

Una mezcla pigmentaria es el resultado de un preparado, de una combinación en la cual se integran diferentes compuestos con el objetivo de obtener un producto con características particulares con el cual realizar un número determinado de pinturas. $\mathrm{Su}$ composición química será diferente, al menos conceptualmente, de toda otra mezcla, incluso si se utilizaran los mismos materiales. En mayor medida, las diferencias serán superiores si variasen las técnicas y los componentes. Lo importante aquí, más allá de los compuestos, son las implicancias históricas que supone el concepto de mezcla pigmentaria, al hacer referencia a un evento de preparación de pintura, que sigue una «receta» particular y que podría ser identificado a partir de los análisis químicos.

En una cueva en donde identificamos motivos de arte rupestre prehispánico en tres tonalidades (rojos, negros y blancos), existen, al menos, tres mezclas pigmentarias. Para este ejemplo no son necesarios los análisis químicos, dado que las diferencias cromáticas apreciables por el ojo humano son suficientes para reconocer las diferencias. Sin embargo, los motivos de un color similar no necesariamente son el producto de la misma mezcla pigmentaria, aun cuando resulten en tonalidades muy próximas. Es allí donde la complejidad se multiplica y los análisis químicos nos permiten observar lo que escapa a nuestros sentidos.

Se analizaron ocho muestras tomadas de los motivos pintados de la Cueva 7 de Oyola. Éstas fueron extraídas utilizando agujas esterilizadas y guantes de látex e introducidas en tubos plásticos Eppendorf. La minúscula cantidad de pintura extraída, de $1 \mathrm{~mm}^{2}$ aprox., hace estos análisis muy poco destructivos. Las muestras fueron analizadas en las instalaciones del Instituto Nacional de Tecnología Industrial y parte de los resultados han sido expuestos en reuniones científicas (Quesada, Gheco, Burgos et al. 2011; Quesada, Gheco, Ybarra et al. 2010). Las técnicas de análisis químico utilizadas fueron: a) Espectroscopía infrarroja por transformada de Fourier (FTIR) ${ }^{6}$. b) Microscopía electrónica de barrido (SEM) y microanálisis elemental por espectroscopía de dispersión de energía de rayos X (EDS) ${ }^{7}$.

La interpretación de los resultados de los análisis químicos es un proceso complejo donde se deben tener en cuenta las posibilidades y límites que ofrecen las técnicas

\footnotetext{
${ }^{6}$ Los análisis fueron realizados con un espectrómetro infrarrojo Nicolet 6700 acoplado a con microscopio infrarrojo Nicolet Centaurus. Los espectros infrarrojos fueron adquiridos mediante dos técnicas: reflexión total atenuada y, microscópicamente, en modo de reflexión-absorción.

7 Los análisis de este trabajo fueron realizados con el microscopio electrónico de barrido del laboratorio de microscopía del Servicio Geológico Minero Argentino.
} 
empleadas. En este sentido, resulta provechoso ocupar algunas líneas para comentar algunos de estos aspectos. En referencia a la espectroscopía infrarroja (FTIR), cada banda del espectro obtenido en los análisis corresponde a un tipo de vibración molecular. Los compuestos orgánicos tienen varias formas de vibrar, por lo que el espectro obtenido tiene distintas bandas, relativamente angostas y bastante características. Esto genera un patrón que resulta muy útil para identificar este tipo de compuestos. Sin embargo, la utilidad de esta técnica en el análisis de compuestos inorgánicos es menor, ya que generan espectros con pocas bandas, en general anchas y similares entre sí, lo que dificulta la caracterización. No obstante, algunos compuestos pueden ser identificados efectivamente mediante espectroscopía infrarroja. Por ejemplo, el carbonato de calcio tiene un espectro con bandas características de la vibración del ion carbonato, en tanto que en el yeso las bandas dadas por los iones sulfato y el agua de cristalización son útiles para su identificación. Por ello fue posible detectar la presencia de estos dos compuestos en las muestras analizadas.

Por otro lado, continuando con esta técnica, debemos tener en cuenta el contenido relativo de los compuestos en las mezclas pigmentarias, ya que, cuanto mayor sea su contenido y su capacidad intrínseca de absorción, más altas serán las posibilidades de detección. Si un compuesto está en una proporción pequeña $(<10 \%)$, puede que no llegue a detectarse en los espectros de la mezcla. Esta situación hace que, en el momento de considerar los resultados obtenidos, no se deba descartar la presencia de otros compuestos que podrían no haber sido identificados mediante FTIR.

En relación con las limitaciones de la técnica de microanálisis elemental (EDS), debemos ser muy cautos a la hora de analizar los valores obtenidos. En primer lugar, ha de señalarse que se trata de una técnica de naturaleza semicuantitativa; asimismo, la mezcla pigmentaria analizada resulta de procesos manuales de elaboración, lo que hace que sea heterogénea en su composición y, así, puedan determinarse porcentajes muy diferentes de los elementos presentes en dos puntos distintos dentro de la misma muestra. Debemos entonces tener en cuenta las precedentes observaciones en el momento de concluir acerca de diferencias encontradas entre las distintas muestras.

A continuación se presentan los resultados obtenidos a partir de la aplicación de las técnicas mencionadas al estudio de las muestras de la cueva Oyola 7, donde se analizan separadamente las pinturas de tonos claros/blancos y las rojas.

\subsection{Pinturas claras/blancas}

Los resultados obtenidos a través del microanálisis elemental (EDS) indican la presencia de una composición cualitativa similar para los elementos hallados en las muestras claras. La intensidad de los picos presentes en los espectros está relacionada con la proporción de cada elemento en la muestra, y puede emplearse para hacer una estimación semicuantitativa de su composición. En líneas generales, podemos decir que los principales elementos presentes en estas pinturas son: carbono (17-24 \% en masa), oxígeno (33-40\%), aluminio (4-11\%), silicio (2-5\%), azufre (4-12\%) y calcio (21-25\%) (Figura 5). En contraposición con las muestras de otros colores, en éstas se destacan valores mayores para el elemento calcio, probablemente debido al uso de una variedad no definida de compuestos calcáreos como tonalizadores. 
Figura 5: Imagen y espectro del análisis EDS de la Muestra OY7M2.

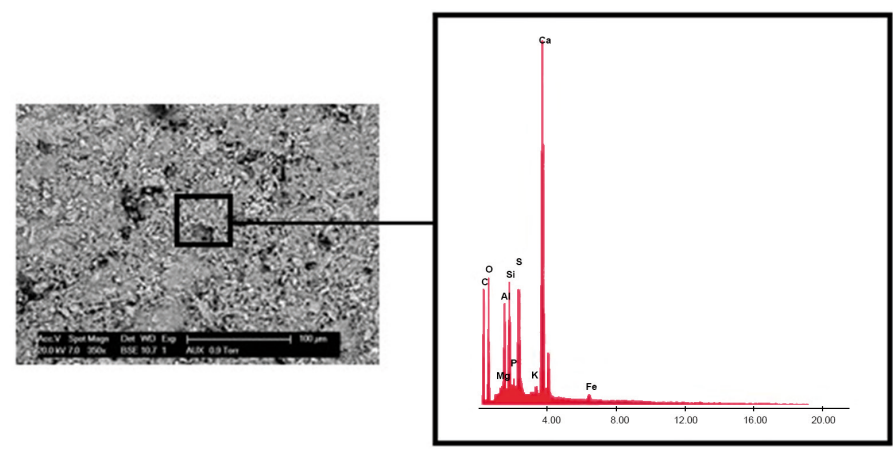

La naturaleza heterogénea de las mezclas pigmentarias se traduce en diferencias apreciables en los porcentajes obtenidos en distintos puntos dentro de las mismas muestras. Esta dispersión de valores dificulta la definición de diferentes mezclas pigmentarias a partir de un criterio basado sólo en las concentraciones elementales en las distintas muestras.

En otro trabajo sobre el arte rupestre de la Sierra de Ancasti (Nazar et al. 2010), los autores interpretan los picos de Al y Si en EDS como el producto de la presencia de arcilla en las muestras analizadas. En el caso de este trabajo, las altas proporciones de $\mathrm{Al}$ y $\mathrm{Si}$ en los espectros EDS podrían ser indicios de que está presente en alguna proporción.

En cuanto a la espectroscopía infrarroja (FTIR), esta técnica nos permite distinguir lo que parecen ser dos mezclas pigmentarias claras/blancas diferentes en cuanto a sus componentes. En primer lugar, parte del patrón de bandas obtenidas en los espectros correspondientes a tres de las cuatro muestras (OY7-1, 0Y7-M2 y OY7-M5 [la OY7M7 no fue analizada mediante FTIR]) se condice con el del yeso $\left(\mathrm{CaSO}_{4} \cdot 2 \mathrm{H}_{2} \mathrm{O}\right.$, sulfato de calcio hidratado), cuyas bandas de absorción se presentan a 3527, 3401, 1683, $1620,1108,1004$ y $667 \mathrm{~cm}^{-1}$ (Figura 6). Por otro lado, los resultados de FTIR revelan la presencia de carbonato de calcio $\left(\mathrm{CaCO}_{3}\right)$, con bandas de absorción a 2512, 1795, 1398,872 y $712 \mathrm{~cm}^{-1}$, sólo en la muestra OY7-M5 (Figura 7). Se aprecia además una banda alrededor de $1035 \mathrm{~cm}^{-1}$, que puede atribuirse a compuestos de tipo silicatos o aluminosilicatos (probablemente arcillas) en las preparaciones de las pinturas.
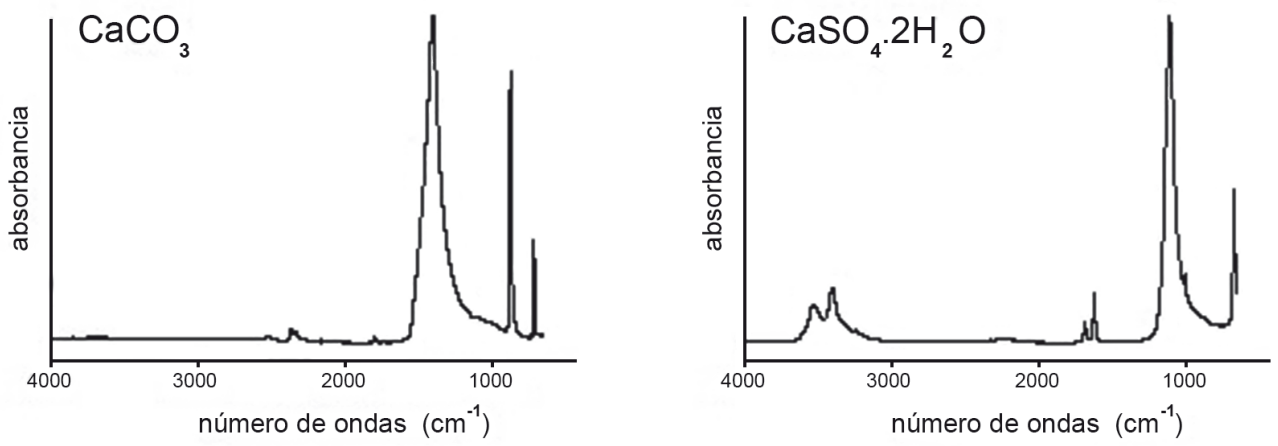

Figura 6: Espectros de carbonato de calcio y yeso de base de datos del INTI. 

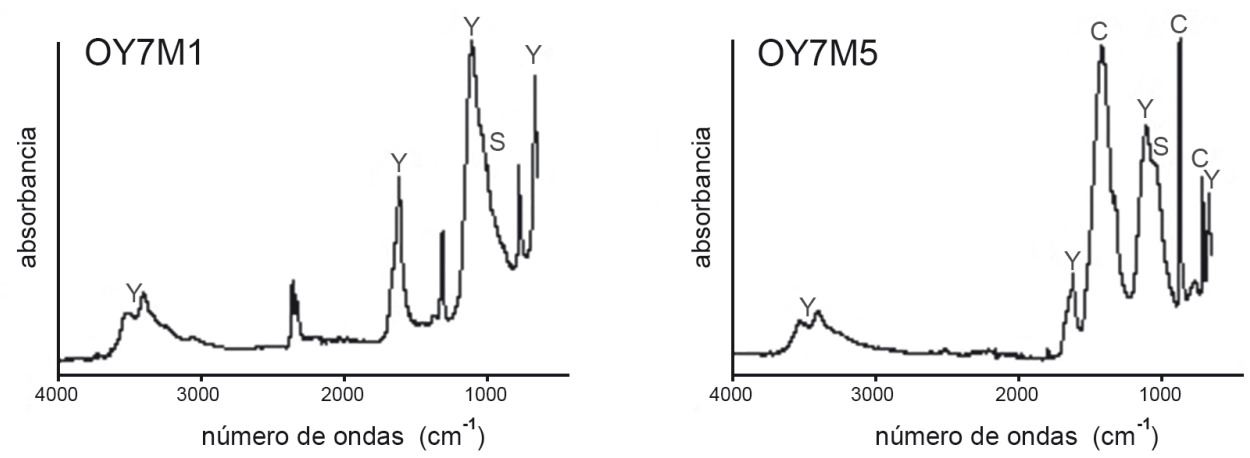

Figura 7: Espectros de dos muestras claras con dos composiciones diferentes; sólo en el espectro de la muestra OY7M5 se observa la presencia de carbonato de calcio. Las bandas indicadas corresponden a yeso (Y), carbonato de calcio (C) y silicatos/aluminosilicatos (S).

De acuerdo con los resultados expresados, entonces, la espectroscopía infrarroja nos permitiría diferenciar dos grupos de pinturas de tonos claros/blancos que se corresponderían con, al menos, dos mezclas pigmentarias. El primer grupo lo componen las muestras OY7M1 y OY7M2, en donde fue detectada sólo la presencia de yeso. El segundo esta representado por la muestra OY7M5 la cual, si bien también posee yeso, sólo se pudo identificar en ella carbonato de calcio.

\subsection{Pinturas rojas}

Por el lado de las pinturas de tonos rojos, el análisis elemental (EDS) indica una mayor concentración de hierro (Fe) en las muestras, lo que se correspondería con el color rojizo de los motivos y con la posibilidad de que se hayan utilizado materiales conteniendo alguna variedad de óxido de hierro $\left(\mathrm{Fe}_{2} \mathrm{O}_{3}\right)$ como cromóforo.

Por otra parte, las diferencias entre los espectros EDS de las pinturas rojas nos permiten hipotetizar sobre la presencia de, al menos, dos mezclas pigmentarias formadas por los grupos de muestras OY7M3 y OY7M4, por un lado, y OY7M6, por otro. La mayor diferencia se aprecia en la concentración de aluminio en la muestra OY7M6 $(21 \% \mathrm{Al})$ que representa casi el doble de la mayor concentración de este elemento obtenida en los análisis de las muestras OY7M3 (12\% Al) y OY7M4 (4\% Al) (Figuras 8 y 9).

En cuanto a los resultados de la espectroscopia infrarroja (FTIR), las bandas se corresponden con la presencia, en todas las muestras, de yeso y silicatos/aluminosilicatos (Figura 10).

\section{Comentarios finales}

Páginas atrás planteamos la posibilidad de comprender algunas cuevas con arte rupestre de la Sierra de El Alto-Ancasti como «obras abiertas», es decir, como espacios cuyos conjuntos pictóricos se habrían modificado con el tiempo a partir del agregado 
Figura 8: Imagen y espectro del análisis EDS de la Muestra OY7M6.

Figura 9: Imagen SEM y espectros de los análisis EDS de la muestra OY7M3.


Figura 10: Espectros infrarrojos de las muestras OY7M3 y OY7M6.

de nuevos motivos a la par que se habrían transformado los significados de las mismas pinturas. De este modo, intentamos continuar una serie de intuiciones que nos permitían sospechar una posible diacronía en la realización de las pictografías. A 
diferencia de los investigadores que abordaron esta problemática en la zona, optamos por no evaluar la adscripción de las pinturas a una u otra cultura, sino que aspiramos a comprender, por otras vías, la historia de las prácticas de pintado que fueron construyendo estos espacios rupestres. Seleccionamos la cueva Oyola 7 para comprobar los alcances y límites de dos técnicas de análisis químicos que nos permitan avanzar en este sentido.

En Oyola 7 relevamos 75 motivos pintados y/o grabados ${ }^{8}$. La documentación detallada de cada uno permitió comprender que, más allá de los cuatro colores identificados en una primera inspección (blanco, rojo, negro y azul [grafitis]), las figuras presentaban matices cromáticos a partir de los cuales se infiere la existencia de mezclas pigmentarias diferentes, aun dentro de lo que parecían colores similares. Así, pudimos diferenciar seis tonos distintos: dos blancos/cremas; dos rojos/naranjas; un negro y un azul (grafitis).

A pesar de la cantidad de motivos relevados, tras un meticuloso examen sólo documentamos diez casos de superposición. Este hecho nos permitió afirmar que las diferentes mezclas pigmentarias halladas en esta cueva fueron realizadas en el transcurso de un período cuya duración aún no conocemos. En líneas generales, los motivos rojos/naranjas fueron superpuestos siempre a los blancos/cremas. A su vez, dentro de éstos últimos, los tonos blancos más claros se sitúan por debajo de los más oscuros o cremas. Sin embargo, los matices cromáticos a los que nos referimos en el párrafo anterior nos hacían sospechar que no todas las pinturas de un mismo color eran el resultado de un mismo evento de preparación y confección de mezclas pigmentarias.

Los análisis químicos brindaron información de gran utilidad. En primer lugar, nos permitieron comenzar a conocer qué materiales fueron utilizados en la realización de las pinturas. En segundo lugar, y más importante para esta discusión, confirmaron la sospecha de que, dentro de los grandes grupos cromáticos en que habíamos clasificado los motivos (blancos, rojos y negros), existían diferentes mezclas pigmentarias. Dentro de los tonos blancos/cremas, los análisis mediante FTIR hicieron posible discriminar dos composiciones diferentes: una con yeso y otra con yeso y carbonato de calcio. Por el lado de las pinturas rojas, los análisis EDS brindaron datos que apuntan a la existencia, cuanto menos, de dos preparaciones diferentes a partir de las variaciones en la concentración de aluminio. Sin embargo, de acuerdo con los alcances y límites de las técnicas de análisis químico que utilizamos, la cantidad de mezclas pigmentarias detectadas se convierte en el número mínimo de pinturas diferentes en Oyola 7.

Es probable que a través de otros métodos analíticos, como la difracción de rayos $\mathrm{X}$, la espectrometría de Raman o la cromatografía gaseosa, podamos profundizar este conocimiento y diferenciar un número mayor de mezclas pigmentarias en las muestras analizadas. La investigación sobre los posibles componentes orgánicos de las mezclas, aspecto aún poco estudiado, podría brindar datos en este sentido e incluso posibilitar futuras dataciones absolutas por AMS.

Como mencionamos, los análisis químicos nos permitieron distinguir dos mezclas pigmentarias blancas/cremas diferentes. Una de ellas, aquella en cuya composición

8 La técnica de grabado suave o raspado se documentó en las inscripciones modernas. 
detectamos únicamente yeso, fue hallada en los motivos 51 y 52 de Oyola 7 (Figura 11). A pesar que los datos químicos nos hacían pensar en una relativa similitud en su composición, ambas figuras presentan tonos cromáticos diferentes e, incluso, se encuentran claramente superpuestas. Estos factores nos mueven a considerar que se podría tratar de distintos eventos de prepara-

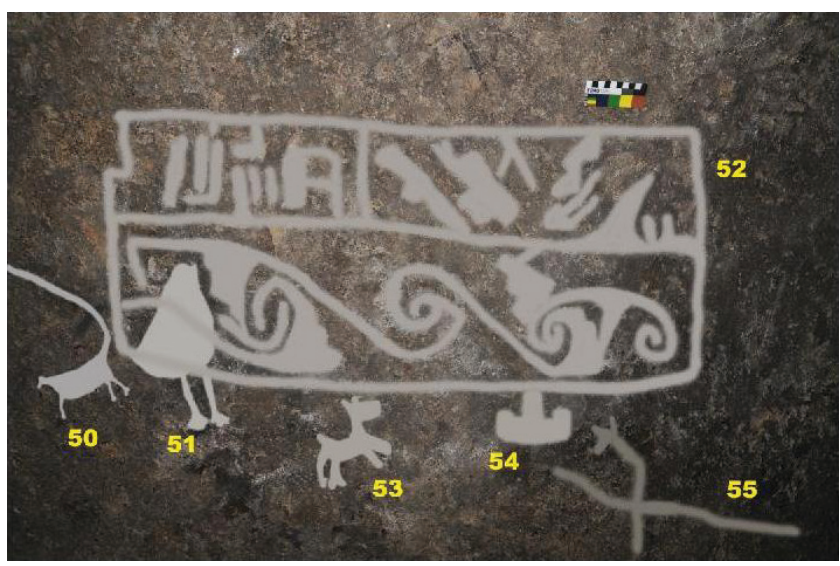

Figura 11: Calcos digitales de los motivos 51 y 52 de Oyola 7. ción de pinturas $y$, a la vez, reforzar la suposición de que la cantidad de mezclas pigmentarias sería mayor que las detectadas mediante los análisis químicos.

En suma, los estudios químicos hicieron posible la distinción de, al menos, cuatro mezclas pigmentarias entre los colores blancos y rojos. Sin embargo, como vimos en el párrafo anterior, las superposiciones nos permiten pensar que dentro de uno de los grupos con una composición química relativamente similar podrían existir dos preparaciones diferentes. A estas cinco mezclas posibles, les debemos agregar la presencia de pinturas negras y azules. Todo ello sumaría un número mínimo de siete mezclas pigmentarias en el interior de Oyola 7, que son equivalentes al mismo número de eventos de preparación de pinturas. Si consideramos que fueron analizadas sólo ocho pinturas de un total de setenta y cinco motivos en la cueva, futuros estudios podrían ampliar estos números.

Aún no conocemos la duración de este proceso ni su ubicación temporal, pero la variedad de pinturas halladas y la presencia de algunas superposiciones entre las figuras nos permiten pensar en una historia, quizás larga, de sucesivos agregados de pinturas. Las dataciones absolutas de los motivos de arte rupestre de la cueva de $\mathrm{La}$ Candelaria, cercana a Oyola, le otorgan al proceso de pintado de aquella cueva una duración de, al menos, 600 años -entre el 700 d.C. y el 1300 d.C.- (Hedges et al. 1998; Llamazares 1999-2000). A partir de los datos expuestos, no sería descabellado pensar en un proceso con una duración similar.

Para finalizar, los datos brindados en este trabajo revelan pistas, indicios, de una historia de sucesivos agregados y modificaciones en las pinturas de un abrigo de la Sierra de Ancasti, en el Noroeste argentino. En tanto «obras abiertas», es posible pensar estas cuevas como espacios cuyos repertorios pictóricos son modificados y reinterpretados en el tiempo. Esto nos informa sobre la complejidad de estos procesos y nos plantea el desafío de buscar alternativas teóricas, metodológicas y técnicas para su estudio. Si bien desconocemos la duración de estos procesos, los grafitis actuales revelan que esta historia aún continua. 
Agradecimientos: Este trabajo fue realizado gracias al apoyo del Fondo Nacional de las Artes, la Secretaría de Ciencia y Tecnología de la Universidad Nacional de Catamarca y el CONICET. Las mejores fotografías incluidas en este trabajo fueron tomadas por Oscar Dechiara. Los posibles errores y omisiones se encuentran bajo nuestra responsabilidad.

\section{Referencias bibliográficas}

\section{Aceñolaza, F., H. Miller y A. Toselli}

1983 «Las rocas cristalinas de la Sierra de Ancasti en el contexto de las Sierras Pampeanas Septentrionales». Münstersche Forschungen Zur Geologie und Paläontologie 59: 251-264.

Aschero, Carlos y María Mercedes Podestá

1986 «El arte rupestre en asentamientos precerámicos de la Puna argentina». Runa 16: 29-57.

BАвот, María del Pilar y María Cristina Apella

2010 «Colores y pigmentos en contexto», en Actas del VIII Simposio Internacional de Arte Rupestre, pp. 15-19. San Miguel de Tucumán.

Bonneau, A., D. Pearce y A. M. Pollard

2012 «A Multi-Technique Characterization and Provenance Study of the Pigments Used in San Rock Art, South Africa». Journal of Archaeological Science 39: 287-294.

De La Fuente, Nicolás R.

1969 «La Cultura de la Aguada: nuevos aportes para su estudio». La Prensa, 23 de noviembre. Buenos Aires.

1979a «Arte rupestre en la región de Ancasti, Provincia de Catamarca», en Actas de las Jornadas de Arqueología del Noroeste Argentino, vol. 2, pp. 408-418. Buenos Aires: Universidad del Salvador.

1979b «Nuevos descubrimientos de arte rupestre en la región de Ancasti, Provincia de Catamarca». Revista del Centro de Estudios de Regiones Secas 1 (2).

1990 «Nuevas pinturas rupestres en la ladera oriental de la Sierra de Ancasti - Catamarca». Revista del Centro de Estudios de Regiones Secas 7: 3-6.

De la Fuente, Nicolás R. y Gloria Arrigoni

1975 «Arte rupestre en la Región Sudeste de la Provincia de Catamarca», en Actas y Trabajos del Primer Congreso de Arqueología Argentina, pp. 177-203. Buenos Aires: Museo Histórico Provincial 'Dr. Julio Marc'.

De la Fuente, Nicolás R. y Adán Roberto Díaz Romero

1974 «Un conjunto de figuras antropomorfas del yacimiento de La Tunita, Provincia de Catamarca». Revista del Instituto de Antropología 5: 35-37. Córdoba.

1979 «Algunos motivos del arte rupestre de la zona de Ancasti (Provincia de Catamarca)», en Miscelánea de arte rupestre de la República Argentina, pp. 37-59. Barcelona: Instituto de Prehistoria y Arqueología, Diputación Provincial.

De la Fuente, Nicolás R., Domingo Carlos Nazar y Eduardo Pelli

2005 «Documentación y diagnóstico del arte rupestre de La Tunita, Provincia de Catamarca, República Argentina», en La Cultura de La Aguada y sus expresiones regionales, pp. 227-244. La Rioja: Universidad Nacional de La Rioja. 
De la Fuente, Nicolás R., Esther Tapia y Jorge Reales

1982 «Nuevos motivos de arte rupestre en la Sierra de Ancasti, Provincia de Catamarca». San Fernando del Valle de Catamarca: Departamento de Educación Prehistoria y Arqueología, Universidad Nacional de Catamarca.

1983 «Otras manifestaciones de arte rupestre en la región de Ancasti, Provincia de Catamarca». San Fernando del Valle de Catamarca: Centro de Investigaciones Antropológicas, Universidad Nacional de Catamarca.

Eco, Umberto

1992 Obra abierta [1962]. Barcelona: Planeta-Agostini.

GHECO, Lucas I.

2011 Una historia en la pared: Hacia una visión diacrónica del arte rupestre de Oyola. Tesis de licenciatura inédita. Universidad Nacional de Catamarca.

Gómez, Marta B. y Nicolás R. De La Fuente

1989 «Arte rupestre en el alero 'El Lechico', Dpto. del Alto, Provincia de Catamarca». Shincal. Revista de la Escuela de Arqueología de Catamarca 1: 25-37.

GonZÁLEZ, Alberto Rex

1961-64 «La Cultura de la Aguada del N.O. Argentino». Revista del Instituto de Antropología 2-3: 205-250. Córdoba.

1977 Arte Precolombino de la Argentina. Buenos Aires: Filmediciones Valero.

1998 Cultura La Aguada. Arqueología y diseños. Buenos Aires: Filmediciones Valero.

Gordillo, Inés y Eva A. CAlomino

2010 «Arte rupestre en el sector septentrional de la Sierra El Alto-Ancasti (Dpto. El Alto, Catamarca)», en Actas del VIII Simposio Internacional de Arte Rupestre, pp. 251-255. San Miguel de Tucumán.

Gramajo, Amalia

2001 Solar de mis mayores. La Concepción del Alto. Santiago del Estero: Ediciones V Centenario.

Gramajo, Amalia y Hugo N. Martínez Moreno

1978 «Otros aportes al arte rupestre del Este catamarqueño». Antiquitas, 26-27: 12-17. Buenos Aires.

Granizo, Alejandra

2010 «Relevamiento del estado de conservación / patologías del arte rupestre», en $J a$ guares, guerreros y serpientes: historia y estética del arte prehispánico de El AltoAncasti. Informe inédito remitido al Fondo Nacional de las Artes, Buenos Aires.

Hedges, Robert E. M., Christopher Bronk Ramsey, Gert J. van Klinken, Paul B. Pettitt; Christina Nielsen-Merch, Alberto Etchegoyen, J. O. Fernández Niello, María Teresa Boschín y Ana María Llamazares

1998 «Methodological Issues in the ${ }^{14} \mathrm{C}$ Dating of Rock Paintings». Radiocarbon 40 (1): $35-44$.

Llamazares, Ana María

1993 «El arte rupestre de los parajes La Tunita y La Toma, ladera oriental de la Sierra de Ancasti, Catamarca». http://www.desdeamerica.org.ar/word/La\%20Tunita\%20 y\%20La\%20Toma.pdf, con acceso el 9/8/2011.

1999-2000 Arte rupestre de la cueva La Candelaria, Provincia de Catamarca. Publicaciones Arqueología 50. Córdoba: Centro de Investigaciones de la Facultad de Filoso- 
fía y Humanidades, Universidad Nacional de Córdoba.

NAZAR, Domingo C.

2003a Parque Arqueológico La Tunita. Puesta en valor integral del arte rupestre de la Sierra de Ancasti, provincia de Catamarca, República Argentina. Tesis de maestría inédita. Universidad Internacional de Andalucía, La Rábida.

2003b Relevamiento arqueológico de la zona austral de la Sierra de Ancasti (Provincia de Catamarca). San Fernando del Valle de Catamarca: CENEDIT, Universidad Nacional de Catamarca.

2010 «Entre el valle, la sierra y la llanura. Una mirada a la problemática Aguada desde el valle de Catamarca». Ponencia presentada en el XVII Congreso Nacional de Arqueología Argentina. Mendoza, 11-15 de octubre.

NAzAr, Domingo C. y Guillermo A. DE LA Fuente

2009 «Parque Arqueológico 'La Tunita'. Una propuesta de protección y puesta en valor del arte rupestre de la cuenca Ipizca-Icaño (Dptos. Ancasti y La Paz, provincia de Catamarca, Argentina)», en Crónicas sobre la piedra. Arte rupestre de las Américas, Marcela Sepúlveda, Luis Briones y Juan Chacama, eds., pp. 47-60. Arica: Ediciones Universidad de Tarapacá.

NAZAR, Domingo C., Lucas I. Gheco y Carlos A. BARot

2010 «El arte rupestre de La Tunita y La Toma, cuenca media e inferior de los ríos Chico y Los Molinos, Departamento Ancasti, Provincia de Catamarca, Argentina», en Actas del VIII Simposio Internacional de Arte Rupestre, pp. 131-135. San Miguel de Tucumán.

QuesAdA, Marcos N. y Lucas I. GHeco

2011 «Modalidades espaciales y formas rituales. Los paisajes rupestres de El Alto-Ancasti». Comechingonia. Revista de Arqueología 15: 63-83.

Quesada, Marcos N., Lucas I. Gheco, Omar Burgos, Andrea Polizsuk y Gabriel Ybarra

2011 «An Investigation of the Rock Art at Oyola Caves». Ponencia presentada en el European Congress and Exhibition on Advanced Materials and Processes (Euromat 2011), Montpellier, 12-15 de septiembre.

Quesada, Marcos N., Lucas I. Gheco, Gabriel Ybarra, Omar Burgos y Andrea Polizsuk

2010 «Hacia una visión diacrónica del arte rupestre de la Sierra de El Alto-Ancasti: el caso de Oyola», en Actas del VIII Simposio Internacional de Arte Rupestre, pp. 136-138. San Miguel de Tucumán.

Segura, Ángel B.

1970 «Pictografías de Catamarca». Boletín de la Junta de Estudios Históricos de Catamarca [1960-68]: 11-33.

1988 El arte rupestre del Este de Catamarca. Las pictografias de la Candelaria. Dpto. Ancasti, Provincia de Catamarca. Catamarca: Editorial Universitaria, Universidad Nacional de Catamarca.

Yacobaccio, Hugo D., M. Paz Cata, Patricia Sola y M. Susana Alonso

2008 «Estudio arqueológico y fisicoquímico de pinturas rupestres en Hornillos 2 (Puna de Jujuy)». Estudios Atacameños 36: 5-28. 